\title{
COMPOSICIÓN QUÍMICA Y EVALUACIÓN DE LA ACTIVIDAD ANTIOXIDANTE DEL ACEITE ESENCIAL FOLIAR DE Eucalyptus camaldulensis DE NORTE DE SANTANDER (COLOMBIA)
}

\section{CHEMICAL COMPOSITION AND EVALUATION OF ANTIOXIDANT ACTIVITY OF LEAF ESSENTIAL OIL Eucalyptus camaldulensis FROM NORTE DE SANTANDER (COLOMBIA)}

\begin{abstract}
Clemente Granados ${ }^{1}$, Gilmar G. Santafé ${ }^{2}$, Diofanor Acevedo ${ }^{3}$
${ }^{1}$ Ingeniero de Alimentos. M.Sc. Universidad de Cartagena, Facultad de Ingeniería, Programa Ingeniería de Alimentos. Campus Piedra de Bolívar, Cartagena - Bolívar, Colombia, e-mail: clementecondeg@gmail.com; ${ }^{2}$ Licenciado en Química y Biología, Ph.D. Ciencias Químicas, Universidad de Córdoba, Facultad de Ciencias Básicas, e-mail: gsantafe@corrreo.unicordoba. edu.co; ${ }^{3}$ Ingeniero de Alimentos. Ph.D. Universidad de Cartagena, Facultad de Ingeniería, Programa Ingeniería de Alimentos. Campus Piedra de Bolívar, Cartagena - Bolívar, Colombia, e-mail: diofanor3000@gmail.com.
\end{abstract}

Rev. U.D.C.A Act. \& Div. Cient. 18(1): 235-240, Enero-Junio, 2015

\section{RESUMEN}

El aceite esencial (AE) foliar de la especie Eucalyptus camaldulensis, recolectada en el departamento de Norte de Santander (Colombia), fue estudiado con el fin de caracterizar su composición química y determinar su actividad antioxidante. La extracción del $\mathrm{AE}$, se realizó por arrastre con vapor y la identificación de los componentes mayoritarios, se hizo por Cromatografía de Gases de Alta Resolución acoplada a espectrometría de masas, para establecer su variabilidad en la composición química, dada las condiciones climáticas que presenta la región. El AE posee mezclas de mono y de sesquiterpenos, siendo el 1,8-cineol, el componente con mayor concentración, con un $81,0 \%$, seguido del $\alpha$-pineno, con un $5,26 \%$. Para precisar la actividad antioxidante de los AE, se usaron dos métodos: capacidad de atrapamiento del catión radical $\mathrm{ABTS}^{+}$y del radical DPPH. El porcentaje de inhibición (\%Inh), con la metodología del radical DPPH, no superó el $10 \%$ y, en los ensayos frente al radical $\mathrm{ABTS}^{+}$, se observó que sólo alcanza valores suficientes a muy altas concentraciones (>250ppm).

Palabras clave: $\mathrm{ABTS}^{+}$radical, componentes aromáticos, $\mathrm{DPPH}$, eucalipto rojo, propiedades antioxidantes, terpenos, terpenoides.

\section{SUMMARY}

The essential leaf oil (AE) of the species Eucalyptus camaldulensis collected in the department of Norte de
Santander (Colombia), was studied in order to characterize its chemical composition and determine their antioxidant activity. The extraction of $\mathrm{AE}$ was performed by steam, and the identification of the main components are made by Gas Chromatography High Resolution coupled to mass spectrometry to determine the chemical composition variability in climatic conditions given that the region presents. The AE has mixtures of mono-and sesquiterpenes, being 1,8-cineole the component with the highest concentration with $81.0 \%$, followed by $\alpha$-pinene with $5.26 \%$. Entrapment capacity ABTS radical cation and radical $\mathrm{DPPH}^{+}$to determine the antioxidant activity of the AE two methods were used. Percent inhibition (\% Inh) with DPPH radical methodology did not exceed $10 \%$ in trials against radical $\mathrm{ABTS}^{+}$was observed that only reaches sufficient values to very high concentrations (>250ppm)

Key words: Radical $\mathrm{ABTS}^{+}$, aromatics, DPPH, red eucalyptus, antioxidant, terpenes, terpenoids.

\section{INTRODUCCIÓN}

Para Ali et al. (2011), el eucalipto es un árbol de la familia Myrtaceae, que incluye 140 géneros y unas 3.800 especies y subespecies, distribuidas en las regiones tropicales y subtropicales. El eucalipto es uno de los géneros más importantes y ampliamente plantados en el mundo. Se trata de un gran árbol de hoja perenne, nativo de Australia y Tasmania e introducido, con éxito, en todo el mundo (Bachir \& Benali, 2012; Tyagi \& Malik, 2011; Elaissi et al. 2012; Shahwar et al. 
2012). En Colombia, se han experimentado varias especies de eucaliptos, siendo las principales $E$. camaldulensis, $E$. teresticornis, E. citriodora, E. globulus y E. grandis (Ospina et al. 2006)

Las especies de eucaliptos son bien conocidas como una fuente rica en aceites esenciales (AE) que, por lo general, se obtienen por medio de vapor o de hidrodestilación (Singh et al. 2012; Marzough et al. 2011). De acuerdo con Tyagi \& Malik, (2011), la composición química y los efectos biológicos de los AE, incluyendo sus propiedades antimicrobianas y potenciales aplicaciones en productos alimenticios, han sido revisadas por diferentes autores (Bakkali et al. 2008).

Los AE son compuestos volátiles, naturales y complejos, caracterizados por un fuerte olor y son formados por las plantas aromáticas, como metabolitos secundarios. Pueden ser sintetizados por todos los órganos de la planta, es decir, brotes, flores, hojas, tallos, ramas, semillas, frutas, raíces, madera o corteza, siendo almacenados en las células secretoras, cavidades, canales, células epidérmicas o tricomas glandulares (Bakkali et al. 2008).

La composición química de los $\mathrm{AE}$ volátiles aislados, a partir de plantas aromáticas depende, en gran medida, del tipo de planta, de la ubicación geográfica, de la temporada de recolección y del método de extracción, entre otras variables (Esen et al. 2007). Para Zeng et al. (2012), los AE se componen, principalmente, de terpenos, de terpenoides y de algunos otros componentes aromáticos y alifáticos, que suelen tener un fuerte olor y bajo peso molecular.

El presente estudio evaluó la capacidad antioxidante del AE foliar del eucalipto $E$. camaldulensis, mediante la utilización de los ensayos $\mathrm{ABTS}^{+}$y de DPPH (2, 2-difenil-1picrilhidracilo).

\section{MATERIALES Y MÉTODOS}

Selección de la materia prima vegetal. La especie conocida comúnmente como eucalipto rojo (E. camaldulensis) fue recolectada en el municipio de Pamplona, vereda Los Animes, en la carretera que conduce a Bucaramanga km 3. Las muestras tomadas pertenecen al material foliar de árboles adultos, de preferencia hojas enteras y verdes. Las hojas fueron recolectadas en la última semana de junio, época normalmente de invierno.

Extracción del AE. Para la extracción del AE, se aplicó la técnica modificada empleada por Stashenko et al. (2004). La obtención del AE, se realizó por destilación con arrastre de vapor, utilizando, para ello, un equipo de hidrodestilación, equipado con manto de calentamiento para balón de $5 \mathrm{~L}$ y trampa de destilación tipo DeanStark. Se tomaron $400 \mathrm{~g}$ del material vegetal, previamente identificado, seleccionado y pesado, que fueron introducidos en el balón y se le adicionó $1 \mathrm{~L}$ de agua; el tiempo de extracción fue de dos horas. El $\mathrm{AE}$ colectado en la trampa de destilación, se separó por decantación y se midió con jeringa de $2 \mathrm{~mL}$, para establecer el rendimiento obtenido, que se calculó mediante la Ecuación 1. El aceite fue deshidratado con $\mathrm{Na}_{2} \mathrm{SO}_{4}$ anhidro y almacenado en viales sellados a $4^{\circ} \mathrm{C}$ hasta su análisis, como lo sugirió Elaissi et al (2011).

$$
\% \text { Rendimiento }=\frac{(m L \text { de aceite esencial })}{\text { Peso en gramos del material vegetal }} * 100
$$

Determinación de las propiedades físicas. $\mathrm{Al} \mathrm{AE}$ obtenido, se le determinó la densidad relativa a $20^{\circ} \mathrm{C}$, con picnómetro de $2 \mathrm{~mL}$; rotación óptica, en polarímetro; índice de refracción, en refractómetro ABBE; punto de ebullición, en tubo de Thiele y solubilidad, en etanol. Para este último, se emplearon soluciones etanólicas de 20,40, 60 y $80 \%$ v/v. De cada una de estas soluciones, se tomaron $100 \mu \mathrm{L}$ y, a cada una, se le agregaron, lentamente, volúmenes de $2 \mu \mathrm{L}$ del $\mathrm{AE}$, hasta que la solución se tornó turbia (punto de saturación).

Análisis de los componentes mayoritarios del AE. Se realizó por cromatografía de gases de alta resolución (CGAR). El equipo utilizado fue un cromatógrafo HP6890A serie II, en interfase, con un detector selectivo de masas HP5973 Network, conectado en línea con un sistema HP-MS ChemStation y la base de datos NIST-2005. Se tomaron $30 \mu \mathrm{L}$ de cada $\mathrm{AE}$ y se disolvieron en $1 \mathrm{~mL}$ de diclorometano; de esta solución, se toma $1,0 \mu \mathrm{L}$ y se inyecta a modo split (10:1). Los espectros de masas (EM), se obtuvieron por ionización electrónica con barrido automático. De acuerdo con Adams (2005), cada EM fue comparado con los estándares de la base de datos y los reportados en la literatura, para obtener los índices de Kovats (IK), relativos a los patrones de alcanos $\mathrm{C}_{8}-\mathrm{C}_{32}$, se realizó el análisis en un cromatógrafo HP 6890A serie II, con detector FID, a $250^{\circ} \mathrm{C}$. Para la identificación y la cuantificación de los componentes de cada $\mathrm{AE}$, se empleó el criterio cromatográfico (IK experimental y teórico) y el criterio espectral (EM). La composición porcentual por CG-FID de cada componente, se analizó por el método de normalización de áreas (Stashenko et al. 2010).

Evaluación de la actividad antioxidante del AE. Se aplicaron dos métodos: capacidad de atrapamiento del catión radical $\mathrm{ABTS}^{+}$y del radical DPPH. De forma exploratoria, se realizaron ensayos preliminares, a una concentración entre 1 y 100ppm para cada muestra de AE y, de acuerdo con los resultados obtenidos, se evaluó la capacidad antioxidante, a concentraciones entre 100 y 300ppm. Para ambos métodos (ABTS y DPPH), se usaron como estándar Trolox y ácido ascórbico, cuya capacidad antioxidante se calculó a las mis- 
mas condiciones de trabajo de las muestras. Los resultados, se expresaron como capacidad antioxidante equivalente al Trolox (TEAC; $\mu$ mol de Trolox/gramos de sustancia evaluada) y capacidad antioxidante equivalente al ácido ascórbico (AAEAC; $\mu$ mol de ácido ascórbico/gramos de sustancia evaluada).

Método del radical DPPH. Se siguió la metodología descrita por Goupy et al. (1999), con modificaciones según Prior et al. (2005). El radical DPPH se disolvió en metanol, para preparar una solución saturada de éste $(80 \mathrm{mg}$ en $5 \mathrm{~mL}$ ), la cual, se almacenó por 24 horas, resguardada de la luz. Transcurrido este tiempo y, a partir de esta solución inicial, se preparó una solución diluida con metanol, hasta una absorbancia de $0,300 \pm 0,05$ a $517 \mathrm{~nm}$.

La absorbancia se midió empleando un espectrofotómetro Génesis 20 Thermospectronic modelo 4001/4 y se calculó el porcentaje de inhibición (\%Inh), usando la Ecuación 2.

$$
\% \text { Inh }=\left[1-\frac{A_{\text {Muestra }}-A_{\text {Blanco }}}{A_{\text {Referencia }}}\right] \times 100
$$

Método del radical $\mathrm{ABTS}^{+}$. Se utilizó el método desarrollado por Re et al. (1999), según modificaciones de Prior et al. (2005), mediante espectroscopía VIS a 734nm.

\section{RESULTADOS Y DISCUSIÓN}

Rendimiento del aceite esencial y propiedades físicas: En la tabla 1 , se muestra el rendimiento obtenido en la extracción del aceite esencial (AE) foliar y las propiedades físicas, medidas para el eucalipto rojo analizado. Allí se puede observar que el rendimiento del $\mathrm{AE}$ foliar del $E$. camaldulensis obtenido en esta investigación es menor al reportado por Yáñez Rueda \& Cuadro Mogollón (2012), para el E. globulus de 4\%, a partir de hojas secas. Gilles et al. (2010) reportan rendimientos entre 2,13 y $3,12 \%$ (w/w), para diferentes especies de Eucalyptus. Barra et al. (2010) obtuvieron rendimientos entre 0,2-0,5\% de $\mathrm{AE}$ del E. camaldulensis Dehnh, valores menores a los aquí obtenidos. Granados et al. (2012) reportan rendimientos entre 1,2 y $0,6 \%$, para el $M$. molis y C. moritzianus, respectivamente, mientras que Siramon \& Ohtani (2007) reportan rendimientos entre 0,83 y 1,63 para el $E$. camaldulensis. Lo anterior, confirma lo manifestado por algunos autores (Gilles et al. 2010; Siramon \& Ohtani, 2007), quienes afirman que el clima, el genotipo, el origen geográfico del crecimiento, las precipitaciones, el régimen de la cosecha y el procedimiento de extracción afectan significativamente el contenido de aceite esencial total de las plantas.

Componentes volátiles mayoritarios del $\mathrm{AE}$. Del perfil cromatográfico del $\mathrm{AE}$ del eucalipto $E$. camaldulensis, obtenido por CGAR-FID, se obtuvieron tres componentes mayoritarios

Tabla 1. Rendimiento y propiedades físicas del AE de Eucaliptus camaldulensis.

\begin{tabular}{|l|c|}
\hline \multicolumn{1}{|c|}{ Análisis } & Resultado \\
\hline Rendimiento del AE & $0,8 \%$ \\
\hline Densidad especifica $20^{\circ} \mathrm{C}$ & $0,91 \mathrm{~g} / \mathrm{ml}$ \\
\hline Índice de refracción $20^{\circ} \mathrm{C}$ & 1,464 \\
\hline Rotación óptica a $20^{\circ} \mathrm{C}$ & $0^{\circ} \mathrm{a}+10^{\circ}$ \\
\hline Solubilidad en etanol & Positiva \\
\hline
\end{tabular}

comunes a los monoterpenos: 1,8-cineol, limoneno y pineno. El promedio de los análisis de los $\mathrm{AE}$ de las muestras foliares mostró que el $\mathrm{AE}$ posee mezclas de mono y de sesquiterpenos, siendo el 1,8-cineol, el componente con mayor concentración, seguido del $\alpha$-pineno, limoneno, $\alpha$-copaeno y Guaiol. Según Ben et al. (2012), los monoterpenos son los principales componentes de los AE de eucalipto. Otros estudios realizados a diferentes especies de eucalipto ratifican lo encontrado en esta investigación (Tabla 2).

Singh et al. (2012), en sus estudios con E. citriodora, hallaron que el aceite, en general, era de naturaleza monoterpenoide, con monoterpenos que constituían un 94,35\% del aceite. Este AE contenía hidrocarburos monoterpenos $(4,49 \%)$, monoterpenoides oxigenados $(89,86 \%)$, hidrocarburos de sesquiterpeno (3,58\%), sesquiterpenos oxigenados $(0,86 \%)$ y otros compuestos no terpenoides $(0,41 \%)$. El principal constituyente del aceite era citronelal $(60,66 \%)$, seguido de $\beta$-citronelol $(12,58 \%)$ y el isopulegol $(8,19 \%)$. Además, también estaban presentes cantidades significativas de $\beta$-cariofileno (2,91\%), $p$-mentano-3, 8-diol (2,87\%), acetato de citronelilo $(2,38 \%)$ y 1,8 -cineol $(1,83 \%)$

Elaissi et al. (2012), luego de trabajar con ocho especies de eucalipto, obtuvieron que el contenido de 1-8 cineol variaba entre $45-70 \%$, el limoneno entre $0,4 \pm 0,2-4,40 \pm 0,3 \%$ y 
Tabla 2. Componentes mayoritarios y concentración (\%) del AE de E. camaldulensis.

\begin{tabular}{|c|c|l|c|c|c|}
\hline No. & \multicolumn{1}{|c|}{$t_{R}$} & \multicolumn{1}{|c|}{ Compuesto } & IK Teórico & IK Exp, & Concentración (\%) \\
\hline 1 & 19,04 & $\alpha$-pineno & 939 & 936 & 5,26 \\
\hline 2 & 22,93 & $\beta$-mirceno & 990 & 992 & 0,50 \\
\hline 3 & 23,92 & $\alpha$-felandreno & 1002 & 1004 & 0,52 \\
\hline 4 & 25,10 & $\alpha$-terpineno & 1018 & 1021 & 1,17 \\
\hline 5 & 25,55 & limoneno & 1029 & 1031 & 2,28 \\
\hline 6 & 26,21 & eucaliptol o 1,8-cineol & 1031 & 1035 & 81,43 \\
\hline 7 & 31,56 & linalol & 1096 & 1098 & 0,32 \\
\hline 8 & 33,87 & terpinen-4-ol & 1177 & 1180 & 1,15 \\
\hline 9 & 35,89 & $\alpha$-terpineol & 1188 & 1190 & 0,51 \\
\hline 10 & 36,66 & $\alpha$-copaeno & 1376 & 1378 & 2,03 \\
\hline 11 & 47,70 & $\beta$-selineno & 1490 & 1498 & 0,59 \\
\hline 12 & 52,10 & guaiol & 1616 & 1621 & 2,21 \\
\hline
\end{tabular}

el $\alpha$-pineno entre $1,0 \pm 0,7-17,6 \pm 7,5 \%$; pero no reportan presencia de limoneno ni guaiol.

Barra et al. (2010), en su estudio con el E. camaldulensis Dehnh, reportan haber encontrado 37 compuestos, los cuales, representan, al menos, el $97 \%$, siendo los principales: p-cimeno (27,8 - 42,7\%); 1,8-cineol (4,1 a 39,5\%); beta-felandreno (3,9-23,8\%); espatulenol $(2,1$ a $15,5 \%)$ y criptone $(3,2$ a $10,2 \%)$.

Sefidkon et al. (2008) estudiaron el efecto del método y el tiempo de destilación en el rendimiento del aceite esencial y la composición de los glóbulos del E. dealbata. Ellos reportaron que con el tiempo, la destilación da lugar a un aumento en el rendimiento y una reducción del contenido de cineol.

Todo lo anterior confirma lo expresado por Fadel et al. (1999), quienes expresaron que el éter monoterpenoide 1,8-cineol, componente típico del aceite de eucalipto, sobre todo en el E. globulus, varía ampliamente. Las variaciones en los ingredientes químicos del $\mathrm{AE}$ de $E$. camaldulensis produce aceites con propiedades químicas diferentes, por lo tanto, los resultados aquí reportados de densidad específica, índice de refracción a $20^{\circ} \mathrm{C}$ y rotación óptica a $20^{\circ} \mathrm{C}$, se presentan sólo a manera de información.
El IKTeérico y el IKExp corresponden a los datos calculados por CGAR-FID en columna HP-5 y, al final, se muestra la concentración relativa, promedio de extracciones realizadas por triplicado.

El aceite posee una moderada cantidad de dos aldehídos no conocidos, cuminal y phellandral (1,4-4,7\%). El aceite esencial fue evaluado en su actividad antifúngica presentando actividad a bajas dosis. La actividad antioxidante ensayada por el test DPPH fue positiva

Evaluación de la actividad antioxidante del AE: Resultados frente al radical DPPH. En la tabla 3, se observa el porcentaje de inhibición (\%Inh), con la metodología del radical $\mathrm{DPPH}$, en ningún caso superó el $10 \%$ y se consideran altos los valores superiores al 30\% (Granados et al. 2012). Se podría decir que en las muestras evaluadas no se encuentran presentes compuestos que puedan estabilizar al DPPH, lo cual, puede explicar la baja actividad mostrada frente a este radical (Granados et al. 2012). Resultados similares obtuvieron Granados et al. (2012), en su estudio con Calycolpus moritzianus y Minthostachys mollis, del Norte de Santander, donde el \%Inh con el método del radical DPPH, en ningún caso superó el $15 \%$.

Tabla 3. Capacidad antioxidante (\%Inh)) frente al radical DPPH de los AE.

\begin{tabular}{|c|c|c|c|c|c|}
\hline & $100 p p m$ & $150 p p m$ & $200 p p m$ & $250 p p m$ & $300 p p m$ \\
\hline \%Inh & 3,20 & 4,01 & 6,16 & 8,14 & 9,02 \\
\hline
\end{tabular}


Trabajos anteriores han estudiado la composición química y la actividad antioxidante del $\mathrm{AE}$ de algunas especies de eucalipto. Barra et al. (2010), en sus investigaciones con AE de partes aéreas de $E$. camaldulensis, hallaron que la actividad antioxidante, evaluada por DPPH, mostró valores entre $0,5 \mathrm{y}$ $5,8 \mathrm{mmol} / \mathrm{L}$. Estos valores son diferentes a los encontrados en esta investigación, porque las composiciones químicas de los eucaliptos son diferentes y varían en función de los diversos orígenes y muestran una fuerte fluctuación durante la fase vegetativa, lo cual, puede explicar que, en especies de eucalipto de otras regiones, sí se presenta una buena capacidad antioxidante frente al radical DPPH.
Evaluación de la actividad antioxidante del AE: Resultados frente al radical catiónico $\mathrm{ABTS}^{+}$. Al realizar el ensayo frente al radical $\mathrm{ABTS}^{+}$, se observó que el \%Inh a 100ppm estuvo muy bajo y solamente alcanza valores suficientes a muy altas concentraciones. En la tabla 4, se puede observar que en ninguno de los casos supera el $40 \%$. Lo anterior confirma que las actividades antioxidantes del p-cimeno, $\alpha$-terpineno y 1,8-cineol son muy pobres (Siramon \& Ohtani, 2007).

Se puede concluir que el AE del Eucalyptus camaldulensis posee mezclas de mono y sesquiterpenos, siendo el 1,8-cineol, el componente con mayor concentración, con un $81 \%$,

Tabla 4. Capacidad antioxidante (\% Inhibición) frente al $\mathrm{ABTS}^{+}$del AE de Eucalyptus camaldulensis.

\begin{tabular}{|c|c|c|c|c|c|}
\hline & $100 p p m$ & $150 p p m$ & $200 p p m$ & $250 p p m$ & $300 p p m$ \\
\hline$\%$ Inh & 19,95 & 21,57 & 38,46 & 38,61 & 38,96 \\
\hline
\end{tabular}

seguido del $\alpha$-pineno, con un 5,26\%. La actividad antioxidante, se obtiene a muy altas concentraciones (>300ppm).

Conflicto de intereses: El manuscrito fue preparado y revisado con la participación de todos los autores, quienes declaramos que no existe ningún conflicto de intereses, que ponga en riesgo la validez de los resultados presentados.

\section{BIBLIOGRAFÍA}

1. ADAMS, R. 2005. Identification of essential oil components by gas chromatography/mass spectroscopy. Allured Publishing Corporation: Carol Stream (USA). 469p.

2. ALI, N.; AHMED, G.; ALI SHAH, S.; SHAH, I.; GHIAS, M.; KHAN, I. 2011. Acute toxicity, brine shrimp cytotoxicity and relaxant activity of fruits of Callistemon citrinus Curtis. BMC Complement Altern Med. 11:99.

3. BACHIR, R.G.; BENALI, M. 2012. Antibacterial activity of the essential oils from the leaves of Eucalyptus globulus against Escherichia coli and Staphylococcus aureus. Asian Pacific J. Trop. Biomed. 2(9):739-742.

4. BAKKALI, F.; AVERBECK, S.; AVERBECK, D.; IDAOMAR, M. 2008. Biological effects of essential oils - A review. Food Chem. Toxicol. 46:446-475.

5. BARRA, A.; CORONEO, V.; DESSI, S.; CABRAS, P.; ANGIONI, A. 2010. Chemical variability, antifungal and antioxidant activity of Eucalyptus camaldulensis essential oil from Sardinia. Nat. Prod. Commun. (United States). 5(2):329-335.
6. BEN, J.; HAOUEL, S.; BOUAZIZ, M.; KHOUJA, M.L. 2012. Seasonal variations in chemical composition and fumigant activity of five Eucalyptus essential oils against three moth pests of stored dates in Tunisia. J. Stored Prod. Res. (United States).48:61-67.

7. ELAISSI, A.; SALAH, B.; MABROUK, S.; CHEMLI, R.; HARZALLAH-SKHIRI, F. 2011. Antibacterial activity and chemical composition of 20 Eucalyptus species essential oils. Food Chem, 129:1427-1434.

8. ELAISSI, A.; ROUIS, Z.; SALEM, N.A.B.; MABROUK, S.; SALEM, Y.B.; SALAH, K.B.H.; AOUNI, M.; FARHAT, F.; CHEMLI, R.; HARZALLAH-SKHIRI, F.; KHOUJA, M.L. 2012. Chemical composition of 8 eucalyptus species essential oils and the evaluation of their antibacterial, antifungal and antiviral activities. BMC Compl. Alter. Med. 12:81.

9. ESEN, G.; AZAZ, A.D.; KURKCUOGLU, M.; CAN BASER, K.H.; TINMAZ, A. 2007. Essential oil and antimicrobial activity of wild and cultivated Origanum vulgare L. subsp. hirtum (Link) letswaart from the Marmara region, Turkey. Flavour Fragr. J.; 22:371-376.

10. FADEL, H.; MARX, F.; EL-SAWY, A.; EL-GHORAB, A. 1999. Effect of extraction techniques on the chemical composition and antioxidant activity of Eucalyptus camaldulensis var. brevirostris leaf oils. Z Lebensm. Unters. Forsch. A. 208:212-216.

11. GILLES, M.; ZHAO, J.; AN, M.; AGBOOLA, S. 2010. Chemical composition and antimicrobial properties 
of essential oils of three Australian Eucalyptus species. Food Chem. 119:731-737.

12. GOUPY, P.; HUGUES, M.; BOIVIN, P.; AMIOT, M.J. 1999. Antioxidant composition and activity of barley (Hordeum vulgare) and malt extracts and of isolated phenolic compounds. J. Sci. Food Agri. 79(12):16251634.

13. GRANADOS, C.; YÁÑEZ, X.; SANTAFÉ, G. 2012. Evaluación de la actividad antioxidante del aceite esencial foliar de Calycolpus moritzianus y Minthostachys mollis, de Norte de Santander. Bistua: Rev. Fac. Cienc. Bás. (Colombia). 10(1):1223.

14. MARZOUG, H.N.B.; ROMDHANE, M.; LEBRIHI, A.; MATHIEU, F.; COUDERC, F.; ABDERRABA, M.; KHOUJA, M.L.; BOUAJILA, J. 2011. Eucalyptus oleosa Essential Oils: Chemical Composition and Antimicrobial and Antioxidant Activities of the Oils from Different Plant Parts (Stems, Leaves, Flowers and Fruits). Molecules, 16(2):1695-1709.

15. OSPINA P., C.M.; HERNÁNDEZ R., R.J.; RODAS P., C.A.; URREGO, J.B.; RIAÑO H., N.M.; ARISTIZABAL V., F.A.; GODOY B., J.A.; OSORIO L., O.I. 2006. Guías silviculturales para el manejo de especies forestales con miras a la producción de madera en la zona andina colombiana. El Eucalipto, Eucalyptus grandis W. Hill ex Maiden. Cenicafé. 52p.

16. PRIOR, R.; WU, X.; SCHAICH, K. 2005. Standardized Methods for the determination of antioxidant capacity and phenolics in foods and dietary supplements. J. Agric. Food Chem. (United States). 53(8):31013113.

17. RE, R.; PELLEGRINI, N.; PROTEGGENTE, A.; PANNALA, A.; YANG, M.; RICE-EVANS, C. 1999. Antioxidant activity applying an improved ABTS radical cation decolorization assay. Free Radical Biol. Medicine. (United States). 26(9-10):1231-1237.

18. SEFIDKON, F.; BAHMANZADEGAN, A.; ASSAREH, M. 2008. Effect of distillation methods and harvesting times on the essential oil and cineole content of Eucalyptus dealbata. Chem. Nat. Compounds. 44(2):250-253.
19. SIRAMON, P.; OHTANI, Y. 2007. Antioxidative and antiradical activities of Eucalyptus camaldulensis leaf oils from Thailand. J. Wood Sci. 53:498-504.

20. SHAHWAR, D.; RAZA, M.A.; BUKHARI, S.; BUKHARI, G. 2012. Ferric reducing antioxidant power of essential oils extracted from Eucalyptus and Curcuma species. Asian Pacific J. Trop. Biomed. Supplement. 2(3):S1633-S1636.

21. SINGH, H.P.; KAUR, S.; NEGI, K.; KUMARI, S.; SAINI, V.; BATISH, D.R.; KOHLI, R.K. 2012. Assessment of in vitro antioxidant activity of essential oil of Eucalyptus citriodora (lemon-scented Eucalypt; Myrtaceae) and its major constituents. LWT - Food Sci. Techn. (UnitedStates). 48(2):237-241.

22. STASHENKO, E.E.; JARAMILLO, B.; MARTÍNEZ, J.R. 2004. Analysis of volatile secondary metabolites from Colombian Xylopia aromatica (Lamarck) by different extraction and headspace methods and gas chromatography. J. Chromatogr. A. 1025:105-113.

23. STASHENKO, E.E.; MARTÍNEZ, J.R.; RUIIZ, C.A.; ARIAS, G.; DURÁN, C.; SALGAR, W.; CALA, M. 2010. Lippia origanoides chemotype differentiation based on essential oil GC-MS and principal component analysis. J. Sep. Sci. (Germany). 33(1):93-103.

24. TYAGI, A.K.; MALIK, A. 2011. Antimicrobial potential and chemical composition of Eucalyptus globulus oil in liquid and vapour phase against food spoilage microorganisms. Food Chem. 126:228-235.

25. YÁÑEZ RUEDA, X.; CUADRO MOGOLLÓN, O.F. 2012. Composición química y actividad antibacteriana del aceite esencial de las especies Eucalyptus globulus y E. camaldulensis de tres zonas de Pamplona (Colombia). Bistua: Rev. Fac. Ciencias Básicas. 10(1):52-61.

26. ZENG, W.; ZHANG, Z.; GAO, H.; JIA, L.R.; HE, Q. 2012. Chemical Composition, Antioxidant, and Antimicrobial Activities of Essential Oil from Pine Needle (Cedrus deodara). J. Food Sci. 77(7):824-829.

Recibido: Febrero 6 de 2014

Aceptado: Abril 7 de 2015

Cómo citar:

Granados, C.; Santafé, G.G.; Acevedo, D. 2015. Composición química y evaluación de la actividad antioxidante del aceite esencial foliar de Eucalyptus camaldulensis de Norte de Santander (Colombia). Rev. U.D.C.A Act. \& Div. Cient. 18(1): 235-240. 